\title{
Noise Performance of the Debuncher Stochastic Cooling Systems
}

A careful measurement of the system noise performance for all 12 Debuncher stochastic cooling systems has been performed. The opportunity to make the measurement was due to a pickup tank warm up to fix a bad preamplifier. A HP power meter and spectrum analyzer were used to measure the noise power and spectral characteristics of each system. Signals were monitored in the tunnel at the medium level transfer switch, before any variable gain devices. Noise power levels observed ranged between -10 to $-30 \mathrm{dBm}$, which is well within the linear calibration range of the power meter. The noise floor of the power meter was measured to be below $-40 \mathrm{dBm}$.

The temperature of the tunnel for the warm measurements was 80 degrees $\mathrm{F}$ or 300 Kelvin. The tanks had been at tunnel temperature for weeks when the warm measurement was made. There was no vacuum in the tanks for the warm measurement. The cold temperature of the tanks at liquid helium was $4.5-5 \mathrm{~K}$. $5 \mathrm{~K}$ was used in the calculations. No component changes were made between the measurements.

The gain of the cryogenic amplifier increases with a decrease in operating temperature. The gain of the cryo amplifier was carefully measured both warm and cold so that this change could be taken into account.

The Noise Figure in $\mathrm{dB}$ and effective noise temperature are derived from the equations below. T2-T1 is the difference in operation temperature in degrees Kelvin, in this case $300-5$ or $295 \mathrm{deg}$. $\mathrm{K}$. Y is the excess noise ratio, which is measured in $\mathrm{dB}$ by the power meter by taking the difference in noise power between warm and cold measurements. This log value must be converted to linear for use in this equation. The value for $\mathrm{Y}$ is also corrected for the increase in gain due to the change in the operating temperature of the amplifier. This data was derived from the warm and cold preamp temperatures measured in the tunnel. The noise figure NF used in the effective noise temperature equation must also be converted to linear. Except for two systems where noise was measured to be below reasonable levels (in red on spreadsheet), the numbers appear to be reasonable.

$$
\text { NoiseFigure }(\mathrm{dB})=10 * \log \left(\frac{\mathrm{T} 2-\mathrm{T} 1 * \mathrm{Y}}{290 *(\mathrm{Y}-1)}+1\right)
$$

\section{EffectiveNoiseTemperature $(\operatorname{degK})=290 *(\mathrm{NF}-1)$}




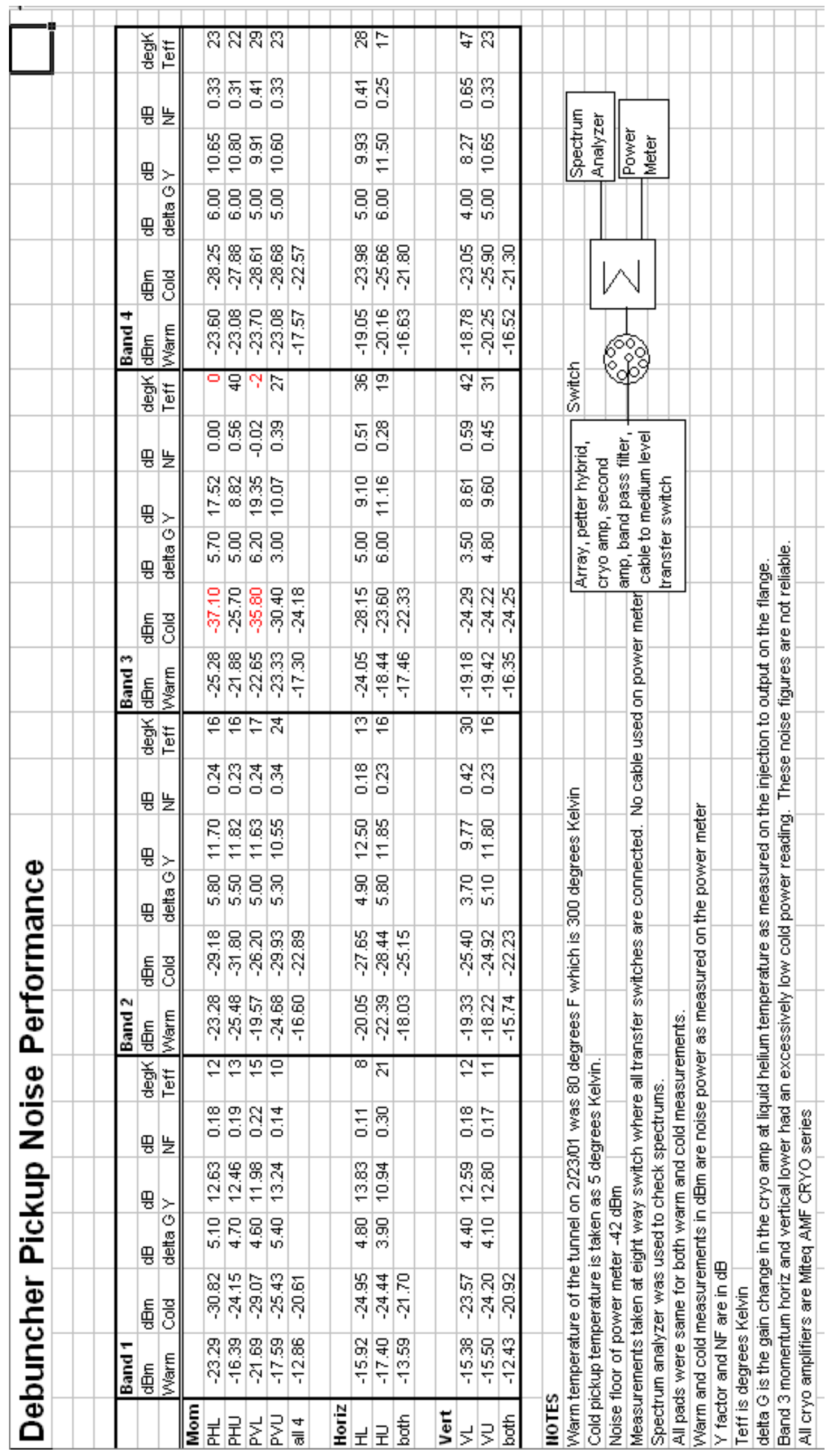

